\title{
APPENDIX: INTERLABORATORY COMPARISON OF LEG 69 AND 70 BASALT STANDARDS1
}

\author{
Hans-W. Hubberten, ${ }^{2}$ Institut für Petrographie und Geochemie der Universität Karlsruhe, Karlsruhe, \\ Federal Republic of Germany \\ and \\ Joan H. Splain, Rosenstiel School of Marine and Atmospheric Science, University of Miami, Miami, Florida
}

To provide an interlaboratory comparison, the laboratories that participated in the analysis of the Leg 69 and 70 basalts were asked to analyze three samples taken from Holes 510 and 504B. Sample IC I was Sample 501-10-1 (69-83 cm), pieces 115 and 116. Sample IC II was Sample 504B-36-1 (91-110 cm), piece 301. Sample IC III was Sample 504B-40-2 $(72-88 \mathrm{~cm})$, piece 532 . All the samples were fine-grained, homogeneous, and relatively fresh in hand specimen. Sample weights exceeded $150 \mathrm{~g}$.

The samples were taken to Karlsruhe, cleaned with distilled water, crushed with help of a hydraulic press, and powdered using an agate mill. The samples were then homogenized and split by using a sample divider. After the samples were divided into eight portions, portions $1,3,5,7$ and $2,4,6,8$ were put together again, forming divisions labeled $\mathrm{A}$ and $\mathrm{B}$. These divisions were divided again into eight portions each, for a total of 16 portions for each sample. One or more of these portions were sent to 12 laboratories, 9 of which returned results. The results for the major elements are presented in Tables 1 to 3 . The nine laboratories (and, where given, publications that give the results of hard-rock analyses made therein) are the Woods Hole Oceanographic Institution (WHOI), the Vernadsky Institute of Geochemistry and Analytical Chemistry, Moscow (VIG, Sharaskin et al., this volume), the Department of Geology and Geography, University of Alabama (ALA), the Centre Océanologique de Bretagne, Brest, France (COB, Etoubleau et al., this volume), the Institut für Petrographie und Geochemie der Universität Karlsruhe, Karlsruhe, Federal Republic of Germany (UKAR, Hubberten et al., this volume), the Department of Geology, University of Newcastle upon Tyne, United Kingdom (UNUT), the Institute of Geology of Ore Deposits, Petrography Mineralogy, and Geochemistry, Moscow (IGOD, Pertsev and Boronikhin, this volume), the Institut Dolomieu, Université de Grenoble, France (UGRE, Laverne and Vivier, in press), and the Centre de Sédimentologie et Géochimie de la Surface, Strasbourg, France (CSGS, Honnorez et al., in press). ${ }^{3}$

\footnotetext{
${ }^{1}$ Cann, J. R., Langseth, M. G., Honnorez, J., Von Herzen, R., P., White, S. M., et al., Init. Repts. DSDP, 69; Washington (U.S. Govt. Printing Office).

2 Present address: Inst. de Geologie, Universidad Autonoma de Nuevo Leon, 67700 Linares, N.L., Mexico.

${ }^{3}$ Analysts and methods used, as given by analysts, are as follows: WHOI-G. Thompson, XRF, ignition; VIG-A. A. Migdisov, chemical, INAA, AAS (preliminary results only); ALA-S. H. Stow, AAS; COB-O. Corre and H. Bougault, XRF, ignition; UKAR-M. Grünhäuser and U. Kramar, XRF, INAA; UNUT-P. J. Oakley; IGOD-G. S. Esikova and S. I. Vronskaya, XRF, AAS; UGRE-G. Vivier, XRF; CSGS-Y. Besnus, spectromètre d'arc.
}

To permit the analyses to be compared, each analysis was recalculated to a dry and reduced state and then normalized to 100 percent. In Tables 4 to 6 these values are presented along with their means and standard deviations (SD). The results of the Vernadsky Institute of Geochemistry (VIG) were not used in calculating the mean and SD because they were sent as preliminary data only.

To present one example of the variations among different laboratories in a different way, the $\mathrm{FeO}^{*}$ and $\mathrm{MgO}$ values obtained on the three standard samples are plotted in Figure 1 as well as the calculated mean.

The homogeneity of the samples can be tested by comparing the analyses for the portions in Divisions A and B of Sample IC I. As can be seen from Table 4, the agreement between IC I A and IC I B is good, with variations that lie within the SD for either. In further discussions the portions in Divisions A and B are therefore considered identical within the uncertainty of analysis.

The results from the different laboratories are in fairly good agreement. However, the standard deviations are larger than in earlier DSDP interlaboratory comparisons (Wright, 1977; Kirkpatrick et al., 1979; Natland et al., 1979 and Staudigel, 1980). There are also systematic deviations from the mean for some of the oxides analyzed by the laboratories (Table 7). The deviations from the mean do not represent deviations from "correct" values, since the calculated mean is based on only 8 analyses. However, they make it clear that care must be taken when data from one laboratory are compared with data from another. Furthermore, they indicate that these data, which were produced as routine analyses, must be considered to have a larger uncertainty than previous data on standard shipboard samples. Differences between different laboratories of $1.5 \%$ in $\mathrm{SiO}_{2}$, $1 \%$ in $\mathrm{Al}_{2} \mathrm{O}_{3}, 1 \%$ in $\mathrm{FeO}^{*}, 0.6 \%$ in $\mathrm{CaO}, 0.5 \%$ in $\mathrm{MgO}$ and $0.5 \%$ in $\mathrm{Na}_{2} \mathrm{O}$ are larger than satisfactory, in our opinion, even for routine analyses.

The trace element data (Tables 8 to 10) show about the same percentages of variation as the major element data (the VIG data are preliminary).

In conclusion, there are systematic differences between the laboratories involved in the investigation of the Costa Rica Rift basalts. The differences are too large to allow comparison of the data from different laboratories for such purposes as detailed discussions of chemical stratigraphy. However, the variations are not large enough to affect the normative classification of the rocks or general elemental variations resulting from such processes as crystal fractionation. 
Table 1. Major element composition of Sample IC I as reported.

\begin{tabular}{|c|c|c|c|c|c|c|c|c|c|c|c|c|}
\hline & \multicolumn{7}{|c|}{ IC I A } & \multicolumn{5}{|c|}{ IC I B } \\
\hline & $\mathrm{COB}$ & UKAR & UNUT & VIG & IGOD & ALA & UGRE & WHOI & VIG & ALA & $\mathrm{COB}$ & CSGS \\
\hline $\mathrm{SiO}_{2}$ & 49.59 & 50.1 & 50.9 & 50.1 & 49.98 & 49.88 & 49.73 & 50.32 & 50.5 & 50.57 & 50.03 & 50.5 \\
\hline $\mathrm{TiO}_{2}$ & 0.75 & 0.75 & 0.81 & 0.82 & 0.77 & 0.83 & 0.82 & 0.77 & 0.82 & 0.90 & 0.75 & 0.70 \\
\hline $\mathrm{Al}_{2} \mathrm{O}_{3}$ & 15.05 & 15.5 & 15.7 & 16.8 & 15.70 & 15.34 & 15.51 & 15.80 & 16.65 & 15.34 & 15.03 & 15.1 \\
\hline $\mathrm{Fe}_{2} \mathrm{O}_{3}$ & 9.60 & 1.83 & 1.91 & 2.46 & 2.42 & 10.42 & 1.89 & - & 2.00 & 10.34 & 9.64 & 9.20 \\
\hline $\mathrm{FeO}$ & - & 7.01 & 6.98 & 6.94 & 6.76 & - & 6.87 & 8.80 & 7.38 & - & - & - \\
\hline $\mathrm{CaO}$ & 13.62 & 13.7 & 13.2 & 13.00 & 13.29 & 13.41 & 12.91 & 13.42 & 12.92 & 13.63 & 13.56 & 13.4 \\
\hline $\mathrm{MgO}$ & 8.33 & 8.39 & 8.26 & 7.62 & 8.35 & 7.78 & 8.12 & 8.60 & 7.60 & 7.99 & 8.12 & 8.00 \\
\hline $\mathrm{MnO}$ & 0.15 & 0.16 & 0.16 & 0.15 & 0.16 & 0.18 & 0.17 & 0.16 & 0.12 & 0.18 & 0.16 & 0.24 \\
\hline $\mathrm{Na}_{2} \mathrm{O}$ & 1.80 & 1.99 & 1.78 & 1.65 & 1.86 & 1.90 & 1.86 & 1.47 & 1.72 & 1.91 & 2.12 & 1.73 \\
\hline $\mathrm{K}_{2} \mathrm{O}$ & 0.02 & 0.02 & 0.03 & 0.02 & 0.06 & 0.03 & 0.06 & 0.01 & 0.03 & 0.03 & 0.02 & $<0.05$ \\
\hline $\mathrm{P}_{2} \mathrm{O}_{5}$ & 0.10 & 0.05 & - & 0.05 & 0.02 & - & - & 0.09 & 0.05 & - & 0.11 & - \\
\hline $\mathrm{H}_{2} \mathrm{O}^{-}$ & 0.21 & - & - & - & 0.47 & - & - & 0.20 & - & - & 0.17 & 0.00 \\
\hline $\mathrm{H}_{2} \mathrm{O}^{+}$ & - & 0.38 & - & 0.70 & 0.31 & 0.56 & 1.33 & - & 0.60 & 0.38 & - & - \\
\hline $\mathrm{CO}_{2}$ & - & 0.18 & - & - & - & - & - & - & - & - & - & - \\
\hline L.0.1. ${ }^{\mathrm{a}}$ & 0.38 & - & - & - & - & - & - & 0.47 & - & - & 0.38 & 0.00 \\
\hline
\end{tabular}

a Loss on ignition.

Table 2. Major element composition of Sample IC II as reported.

\begin{tabular}{|c|c|c|c|c|c|c|c|c|c|}
\hline & \multicolumn{7}{|c|}{ IC $\| \mathrm{A}$} & \multicolumn{2}{|c|}{ IC II B } \\
\hline & WHOI & VIG & ALA & $\mathrm{COB}$ & UKAR & UNUT & IGOD & UGRE & CSGS \\
\hline 2 & 50.72 & 50.2 & 50.31 & 49.99 & 50.0 & 51.0 & 49.55 & 49.76 & 0.3 \\
\hline & 0.95 & 0.99 & 1.06 & & & & & 1.03 & 0.84 \\
\hline $\mathrm{Al}_{2} \mathrm{O}_{3}$ & 15.41 & 15.9 & 14.98 & 14 & 15.0 & 15. & 15 & 17 & 14.5 \\
\hline $\mathrm{Fe}_{2} \mathrm{O}_{3}$ & & 2.62 & 11.10 & 10.53 & 2.3 & 2.30 & 3.03 & 2.31 & 10.0 \\
\hline $\mathrm{FeC}$ & 9.50 & 7.34 & M.No & - & 7.25 & 7.04 & & 7.09 & - \\
\hline $\mathrm{CaO}$ & 12.47 & 12.33 & 12.19 & 12.65 & 12.6 & 12.1 & 12.23 & 11.91 & 12.4 \\
\hline $\mathrm{MgO}$ & 8.58 & 7.70 & 7.87 & 8.58 & 8.32 & 8.27 & 8.42 & 8.15 & 7.94 \\
\hline $\mathrm{Mn}$ & 0. & 0.12 & 0.18 & 0. & 0. & 0.17 & 0.17 & 0.17 & 0.25 \\
\hline $\mathrm{Na}_{2}$ & 1.79 & 2.01 & 2.17 & 2.00 & 2.31 & 2.0 & 2. & 2.13 & 1.94 \\
\hline $\mathrm{K}_{2} \mathrm{O}$ & $0.00_{2}$ & 0.02 & 0.03 & 0.01 & 0.01 & 0.02 & 0. & 0.12 & $<0.05$ \\
\hline $\mathrm{P}_{2} \mathrm{O}_{5}$ & 0.11 & 0.07 & - & 0.13 & 0.06 & - & 0.0 & - & - \\
\hline $\mathrm{H}_{2} \mathrm{O}$ & 0.57 & - & $=$ & 0.43 & - & 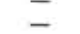 & 0.4 & $=$ & 0.28 \\
\hline $\mathrm{H}_{2} \mathrm{O}$ & - & 0.76 & 1.07 & 0.40 & 0. & $=$ & 0.84 & 1.90 & \\
\hline & $=$ & - & - & - & 0.1 & 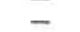 & & W. & \\
\hline L.O.I. ${ }^{a}$ & 0.61 & $\overline{-}$ & $\overline{-}$ & 0.77 & -1 & - & - & $\overline{-}$ & \\
\hline
\end{tabular}

${ }^{a}$ Loss on ignition.
Table 3. Major element composition of Sample IC III as reported.

\begin{tabular}{|c|c|c|c|c|c|c|c|c|c|}
\hline & \multicolumn{8}{|c|}{ IC III A } & \multirow{2}{*}{$\frac{\text { IC III B }}{\operatorname{csGS}}$} \\
\hline & wHOI & VIG & ALA & COB & UKAR & UNUT & IGOD & UGRE & \\
\hline & 49.70 & 49.5 & 48.85 & 49.42 & 49.0 & 50.75 & 48.95 & 48.56 & 49.7 \\
\hline & 0.81 & 0.87 & $\begin{array}{r}4.63 \\
0.89\end{array}$ & $\begin{array}{r}47.422 \\
0.79\end{array}$ & 0.78 & & 0.82 & 0.83 & 0.72 \\
\hline $\mathrm{Al}_{2} \mathrm{O}_{3}$ & $\begin{array}{l}1.060 \\
16.80\end{array}$ & 17.50 & 16.18 & 15.76 & 16.2 & 16.35 & 16.28 & 16.53 & 15.4 \\
\hline $\mathrm{Fe}_{2} \mathrm{O}_{3}$ & & 2.16 & 9.91 & 9.37 & 1.89 & 1.65 & 2.61 & 1.93 & 8.9 \\
\hline & 8.49 & 6.79 & & - & 6.64 & 6.79 & 6.27 & 6.62 & \\
\hline $\mathrm{CaO}$ & 12.99 & 12.33 & 13.17 & 13.13 & 13.2 & 12.6 & 12.77 & 12.47 & 12.9 \\
\hline $\mathrm{MgO}$ & 8.99 & 8.00 & 8.10 & 8.78 & 8.78 & 8.57 & 8.74 & 8.56 & 8.23 \\
\hline $\mathrm{MnO}$ & 0.15 & 0.11 & 0.17 & 0.16 & 15 & 0.16 & 0.15 & 0.16 & 0.23 \\
\hline $\mathrm{Na}_{2} \mathrm{O}$ & 1.67 & 1.89 & 1.99 & 1.96 & 2.09 & 1.87 & 2.04 & 1.92 & 1.78 \\
\hline $\mathrm{K}_{2} \mathrm{O}$ & 0.006 & 0.02 & 0.01 & 0.02 & 0.01 & 0.02 & 0.08 & 0.04 & $<0.05$ \\
\hline $\mathrm{P}_{2} \mathrm{O}_{5}$ & 0.09 & 0.07 & - & 0.08 & 0.03 & - & 0.03 & - & \\
\hline $\mathrm{H}_{2} \mathrm{O}$ & 0.45 & - & - & 0.37 & - & - & 0.43 & - & 0.00 \\
\hline $\mathrm{H}_{2} \mathrm{O}$ & - & 0.71 & 1.00 & - & 0.68 & - & 0.79 & 1.83 & \\
\hline Co & - & & & - & 0.14 & - & - & - & \\
\hline L.O. $\mathrm{I}^{\mathrm{a}}$ & 0.73 & - & - & 0.77 & - & - & - & - & 0.58 \\
\hline
\end{tabular}

a Loss on ignition.

Table 4. Major element composition of Sample IC I when recalculated to a dry and reduced state and normalized to 100 percent.

\begin{tabular}{|c|c|c|c|c|c|c|c|c|c|c|c|c|c|c|c|c|}
\hline & \multicolumn{8}{|c|}{ IC I A } & \multicolumn{6}{|c|}{ IC I B } & \multicolumn{2}{|c|}{ IC $1 \mathrm{~A}+\mathrm{IC} \mathrm{I} \mathrm{B}$} \\
\hline & $\mathrm{COB}$ & UKAR & UNUT & VIGa & IGOD & ALA & UGRE & Mean & WHOI & VIG & ALA & $\mathrm{COB}$ & CSGS & Mean & Mean & SD \\
\hline $\mathrm{SiO}_{2}$ & 50.58 & 50.44 & 51.14 & 50.42 & 50.42 & 50.52 & 50.87 & 50.66 & 50.65 & 50.71 & 50.64 & 50.75 & 51.53 & 50.89 & 50.65 & 0.36 \\
\hline $\mathrm{TiO}_{2}$ & 0.76 & 0.76 & 0.81 & 0.83 & 0.78 & 0.84 & 0.84 & 0.80 & 0.78 & 0.82 & 0.90 & 0.76 & 0.71 & 0.79 & 0.79 & 0.05 \\
\hline $\mathrm{Al}_{2} \mathrm{O}_{3}$ & 15.35 & 15.61 & 15.77 & 16.91 & 15.84 & 15.54 & 15.87 & 15.66 & 15.90 & 16.72 & 15.36 & 15.25 & 15.41 & 15.48 & 15.59 & 0.24 \\
\hline $\mathrm{FeO}$ & 8.81 & 8.72 & 8.74 & 9.21 & 9.02 & 9.50 & 8.77 & 8.93 & 8.86 & 9.22 & 9.32 & 8.81 & 8.45 & 8.86 & 8.90 & 0.31 \\
\hline $\mathrm{CaO}$ & 13.89 & 13.79 & 13.26 & 13.08 & 13.41 & 13.58 & 13.21 & 13.52 & 13.51 & 12.97 & 13.65 & 13.76 & 13.67 & 13.65 & 13.57 & 0.22 \\
\hline $\mathrm{MgO}$ & 8.50 & 8.45 & 8.30 & 7.67 & 8.42 & 7.88 & 8.31 & 8.31 & 8.56 & 7.63 & 8.00 & 8.24 & 8.16 & 8.24 & 8.28 & 0.22 \\
\hline $\mathrm{MnO}$ & 0.15 & 0.16 & 0.16 & 0.15 & 0.16 & 0.18 & 0.17 & 0.16 & 0.16 & 0.12 & 0.18 & 0.16 & 0.24 & 0.18 & 0.17 & 0.03 \\
\hline $\mathrm{Na}_{2} \mathrm{O}$ & 1.84 & 2.00 & 1.79 & 1.66 & 1.88 & 1.92 & 1.90 & 1.89 & 1.48 & 1.73 & 1.91 & 2.15 & 1.76 & 1.82 & 1.86 & 0.17 \\
\hline $\mathrm{K}_{2} \mathrm{O}$ & 0.02 & 0.02 & 0.03 & 0.02 & 0.06 & 0.03 & 0.06 & 0.04 & 0.01 & 0.03 & 0.03 & 0.02 & 0.05 & 0.02 & 0.03 & 0.02 \\
\hline $\mathrm{P}_{2} \mathrm{O}_{5}$ & 0.10 & 0.05 & - & 0.05 & 0.02 & - & - & 0.06 & 0.09 & 0.05 & - & 0.11 & - & 0.10 & 0.07 & 0.04 \\
\hline Total & 100.00 & 100.00 & 100.00 & 100.00 & 100.01 & 99.99 & 100.00 & - & 100.00 & 100.00 & 99.99 & 100.01 & 99.98 & - & - & - \\
\hline
\end{tabular}

a Values were not used to calculate means and standard deviations.

\section{REFERENCES}

Honnorez, J., Karpoff, A.-M., and Trauth-Badaut, D., in press. Sedimentology, mineralogy, and geochemistry of green clay samples from the Galapagos hydrothermal mounds, Holes 506, 506C, and 507D, Deep Sea Drilling Project Leg 70 (preliminary data). In Honnorez, J., Von Herzen, R. P., et al., Init. Repts. DSDP, 70: Washington (U.S. Govt. Printing Office).

Kirkpatrick, R. J., and the Leg 46 Shipboard Scientific Party, 1979. Interlaboratory comparison of Leg 46 basalt standards. In Dmitriev, L., Heirtzler, J., et al., Init. Repts. DSDP, 46: Washington (U.S. Govt. Printing Office), 293-297.

Laverne, C., and Vivier, G., in press. Petrological and chemical study of basement basalts from the Galapagos Spreading Center, Leg 70 .
In Honnorez, J., Von Herzen, R. P., et al., Init. Repts. DSDP, 70: Washington (U.S. Govt. Printing Office).

Natland, J., Bougault, H., Fujii, T., Graham, A. L., Melson, W. G., Prosser, E., Rhodes, J. M., and Zolotarev, B., 1979. Chemical data for Sites 395 and 396 analytical procedures and comparison of interlaboratory standards. In Melson, W. G., Rabinowitz, P. D., et al., Init. Repts. DSDP, 45: Washington (U.S. Govt. Printing Office), 681-705.

Staudigel H., 1980. Chemical analyses of interlaboratory standards, In Donnelly, T., Francheteau, J., Bryan, W., Robinson, P., Flower, M., Salisbury, M., et al., Init. Repts. DSDP, 51, 52, 53, Pt. 2: Washington (U.S. Govt. Printing Office), 1331-1333.

Wright, T. L., 1977. Chemical analyses of interlaboratory standards, In Aumento, F., Melson, W. G., et al., Init. Repts. DSDP, 37: Washington (U.S. Govt. Printing Office), 367-370. 
Table 5. Major element composition of Sample IC II when recalculated to a dry and reduced state and normalized to 100 percent.

\begin{tabular}{|c|c|c|c|c|c|c|c|c|c|c|c|}
\hline & \multicolumn{7}{|c|}{ IC II A } & \multicolumn{2}{|c|}{ IC II B } & \multicolumn{2}{|c|}{$\mathrm{IC} \| \mathrm{A}+\mathrm{B}$} \\
\hline & WHOI & vIGa & ALA & $\mathrm{COB}$ & UKAR & UNUT & IGOD & UGRE & CSGS & Mean & SD \\
\hline $\mathrm{SiO}_{2}$ & 50.87 & 50.69 & 50.93 & 50.70 & 50.62 & 51.54 & 50.30 & 50.98 & 51.74 & 50.96 & 0.47 \\
\hline $\mathrm{TiO}_{2}^{2}$ & 0.95 & 1.00 & 1.07 & 0.94 & 0.92 & 0.97 & 0.95 & 1.06 & 0.86 & 0.96 & 0.07 \\
\hline $\mathrm{Al}_{2} \mathrm{O}_{3}$ & 15.46 & 16.05 & 15.17 & 14.88 & 15.19 & 15.46 & 15.59 & 15.54 & 14.92 & 15.28 & 0.28 \\
\hline $\mathrm{FeO}^{*}$ & 9.53 & 9.79 & 10.11 & 9.61 & 9.51 & 9.21 & 9.76 & 9.39 & 9.26 & 9.55 & 0.29 \\
\hline $\mathrm{CaO}$ & 12.51 & 12,45 & 12.34 & 12.83 & 12.76 & 12.23 & 12.41 & 12.20 & 12.75 & 12.50 & 0.25 \\
\hline $\mathrm{MgO}$ & 8.61 & 7.77 & 7.97 & 8.70 & 8.42 & 8.36 & 8.55 & 8.35 & 8.17 & 8.39 & 0.23 \\
\hline $\mathrm{MnO}$ & 0.17 & 0.12 & 0.18 & 0.16 & 0.17 & 0.17 & 0.17 & 0.17 & 0.26 & 0.18 & 0.03 \\
\hline $\mathrm{Na}_{2} \mathrm{O}$ & 1.80 & 2.03 & 2.20 & 2.03 & 2.34 & 2.05 & 2.18 & 2.18 & 1.99 & 2.10 & 0.16 \\
\hline $\mathrm{K}_{2} \mathrm{O}$ & 0.002 & 0.02 & 0.03 & 0.01 & 0.01 & 0.02 & 0.05 & 0.12 & 0.05 & 0.04 & 0.04 \\
\hline $\mathrm{P}_{2} \mathrm{O}_{5}$ & 0.11 & 0.07 & - & 0.13 & 0.06 & - & 0.03 & - & - & 0.08 & 0.05 \\
\hline Total & 100.01 & 99.99 & 100.00 & 99.99 & 100.00 & 100.01 & 99.99 & 99.99 & 100.00 & - & - \\
\hline
\end{tabular}

a Values were not used to calculate means and standard deviations.

Table 6. Major element composition of Sample IC III when recalculated to a dry and reduced state and normalized to 100 percent.

\begin{tabular}{|c|c|c|c|c|c|c|c|c|c|c|c|}
\hline & \multicolumn{8}{|c|}{ IC III A } & \multirow{2}{*}{$\frac{\text { IC III B }}{\text { CSGS }}$} & \multicolumn{2}{|c|}{ IC III A + B } \\
\hline & WHOI & VIG $^{a}$ & ALA & $\mathrm{COB}$ & UKAR & UNUT & IGOD & UGRE & & Mean & SD \\
\hline $\mathrm{O}_{2}$ & 49.85 & 49.99 & 49.70 & 50.16 & 49.71 & 51.04 & 49.71 & 49.84 & 51.23 & 50.15 & 0.62 \\
\hline $\mathrm{TiO}_{2}$ & 0.81 & 0.88 & 0.91 & 0.80 & 0.79 & 0.84 & 0.83 & 0.85 & 0.74 & 0.82 & 0.11 \\
\hline $\mathrm{Al}_{2} \overline{\mathrm{O}}_{3}$ & 16.85 & 17.67 & 16.46 & 16.00 & 16.43 & 16.44 & 16.53 & 16.97 & 15.87 & 16.44 & 0.37 \\
\hline $\mathrm{FeO} 0^{*}$ & 8.52 & 8.82 & 9.08 & 8.56 & 8.46 & 8.33 & 8.75 & 8.58 & 8.26 & 8.57 & 0.26 \\
\hline $\mathrm{CaO}$ & 13.03 & 12.45 & 13.40 & 13.33 & 13.39 & 12.67 & 12.97 & 12.80 & 13.30 & 13.11 & 0.28 \\
\hline $\mathrm{MgO}$ & 9.02 & 8.08 & 8.24 & 8.91 & 8.91 & 8.62 & 8.87 & 8.79 & 8.48 & 8.73 & 0.26 \\
\hline $\mathrm{MnO}$ & 0.15 & 0.11 & 0.17 & 0.16 & 0.15 & 0.16 & 0.15 & 0.16 & 0.24 & 0.16 & 0.03 \\
\hline $\mathrm{Na}_{2} \mathrm{O}$ & 1.68 & 1.91 & 2.02 & 1.99 & 2.12 & 1.88 & 2.07 & 1.97 & 1.84 & 1.95 & 0.14 \\
\hline $\mathrm{K}_{2} \mathrm{O}$ & 0.006 & 0.02 & 0.01 & 0.02 & 0.01 & 0.02 & 0.08 & 0.04 & 0.05 & 0.03 & 0.03 \\
\hline $\mathrm{P}_{2} \mathrm{O}_{5}$ & 0.09 & 0.07 & - & 0.08 & 0.03 & - & 0.03 & - & - & 0.06 & 0.02 \\
\hline Total & 100.00 & 100.00 & 99.99 & 100.01 & 100.00 & 100.00 & 99.99 & 100.00 & 100.01 & - & - \\
\hline
\end{tabular}

a Values were not used to calculate means and standard deviations.

I C I

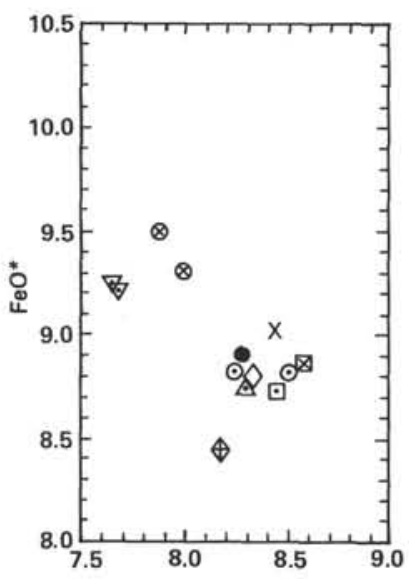

IC II

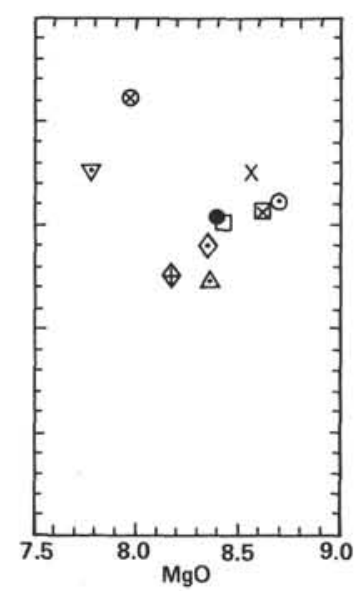

IC III

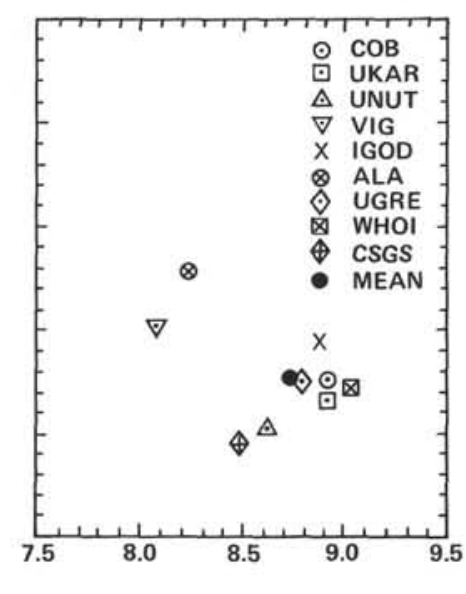

Figure 1. $\mathrm{FeO}^{*}-\mathrm{MgO}$ plot of the recalculated values from Tables 4 to 6 for the three Leg 69 and 70 samples. 
Table 7. Deviations from the mean value for all major elements for the different laboratories.

\begin{tabular}{|c|c|c|c|c|c|c|c|c|c|c|}
\hline & & csGs & $\mathrm{COB}$ & UKAR & UNUT & VIG & IGOD & ALA & UGRE & WHOI \\
\hline \multirow{3}{*}{$\mathrm{SiO}_{2}$} & IC & +0.88 & 0.10 & -0.21 & +0.49 & -0.23 & -0.23 & -0.13 & +0.22 & \pm 0.00 \\
\hline & & & & & & & & -0.02 & & \\
\hline & IC III & +1.08 & +0.01 & -0.41 & +0.89 & -0.16 & -0.41 & -0.45 & -0.31 & -0.30 \\
\hline \multirow[t]{3}{*}{$\mathrm{TiO}_{2}$} & IC I & -0.08 & -0.03 & -0.03 & +0.02 & +0.04 & -0.01 & +0.05 & +0.05 & -0.01 \\
\hline & IC II & -0.10 & -0.02 & -0.04 & +0.01 & +0.04 & -0.01 & +0.11 & +0.10 & -0.01 \\
\hline & IC III & -0.08 & -0.02 & -0.03 & +0.02 & +0.06 & +0.01 & +0.09 & +0.03 & -0.01 \\
\hline \multirow[t]{3}{*}{$\mathrm{Al}_{2} \mathrm{O}_{3}$} & IC 1 & -0.18 & -0.35 & +0.02 & +0.18 & +1.32 & +0.25 & -0.05 & +0.28 & +0.31 \\
\hline & IC II & -0.36 & -0.40 & -0.09 & +0.18 & +0.77 & +0.31 & -0.11 & +0.26 & +0.18 \\
\hline & IC III & -0.57 & -0.44 & -0.01 & \pm 0.00 & +1. & +0.09 & +0.02 & +0.53 & +0.41 \\
\hline \multirow[t]{3}{*}{$\mathrm{FeO}$} & ic 1 & -0.45 & -0.09 & -0.18 & -0.16 & +0.31 & +0.12 & +0.60 & -0.13 & -0.04 \\
\hline & IC II & -0.29 & +0.06 & -0.04 & -0.34 & +0.24 & +0.21 & +0.56 & -0.16 & -0.02 \\
\hline & IC III & -0.31 & +0.18 & -0.1 & -0.24 & +0.25 & +0.18 & +0.51 & +0.01 & -0.05 \\
\hline \multirow[t]{3}{*}{$\mathrm{CaO}$} & IC I & +0.10 & +0.32 & +0.22 & -0.31 & -0.49 & -0.16 & +0.01 & -0.36 & -0.06 \\
\hline & IC II & +0.25 & +0.33 & +0.26 & -0.27 & -0.05 & -0.09 & -0.16 & -0.30 & +0.01 \\
\hline & IC III & +0.19 & +0.22 & +0. & -0.44 & -0.68 & -0.14 & +0.29 & -0.31 & -0.08 \\
\hline \multirow[t]{3}{*}{$\mathrm{MgO}$} & IC I & -0.12 & +0.22 & +0.17 & +0.02 & -0.61 & +0.14 & -0.40 & +0.03 & +0.28 \\
\hline & IC II & -0.22 & +0.31 & +0.03 & -0.03 & -0.62 & +0.16 & -0.42 & -0.04 & +0.22 \\
\hline & IC II & -0.25 & +0 & +0 & -0 & -0.65 & & -0.49 & & +0.29 \\
\hline \multirow[t]{3}{*}{$\mathrm{MnO}$} & IC I & +0.07 & -0.02 & -0.01 & -0.01 & -0.02 & -0.01 & +0.01 & \pm 0.00 & -0.01 \\
\hline & IC II & +0.08 & -0.02 & -0.01 & -0.01 & -0.06 & -0.01 & \pm 0.00 & -0.01 & -0.01 \\
\hline & IC III & +0. & \pm 0 . & -0.01 & \pm 0.00 & -0.05 & -0.01 & +0.01 & & -0.01 \\
\hline \multirow[t]{3}{*}{$\mathrm{Na}_{2} \mathrm{O}$} & IC 1 & -0.10 & -0.02 & +0.14 & -0.07 & -0.20 & +0.02 & +0.06 & +0.04 & -0.18 \\
\hline & IC II & -0.1 & -0.07 & +0 & -0 & -0 & +0 & +0.10 & $+c$ & -0.30 \\
\hline & ic il & -0.11 & +0.04 & +0.17 & -0.07 & -0 & +0.12 & +0.07 & +0.02 & -0.27 \\
\hline \multirow[t]{3}{*}{$\mathrm{K}_{2} \mathrm{O}$} & IC 1 & +0.02 & -0.01 & -0.01 & \pm 0.00 & -0.01 & +0.03 & \pm 0.00 & +0.03 & -0.02 \\
\hline & IC & & & & -0 & -0 & & -0.01 & +0.08 & -0.04 \\
\hline & IC III & +0.02 & -0.01 & -0.02 & -0.01 & -0.04 & +0.05 & -0.02 & +0.01 & -0.02 \\
\hline
\end{tabular}

Table 8. Trace elements in Sample IC I as reported.

\begin{tabular}{lcccccccc}
\hline & VIG & UKAR & COB & WHOI & ALA & UGRE & IGOD & CSGS \\
\hline $\mathrm{Rb}$ & 6 & - & 1.24 & 1.3 & - & $<10$ & 4.6 & - \\
$\mathrm{Sr}$ & 52 & 59 & 51 & 43.5 & - & 42 & 58 & 48 \\
$\mathrm{Ba}$ & - & - & - & $<20$ & - & 11 & 22.4 & 2 \\
$\mathrm{Li}$ & 5 & - & - & - & 8.1 & - & 4.6 & - \\
$\mathrm{Y}$ & - & 23 & 24 & 22.6 & - & - & - & 20 \\
$\mathrm{Zr}$ & - & 31 & 38 & 50.9 & - & - & - & 36 \\
$\mathrm{Co}$ & 42 & 43 & - & 46.3 & 35.7 & 69 & 38 & 46 \\
$\mathrm{Ni}$ & 72 & 77 & 83.6 & 91.8 & 86 & 120 & 74 & 107 \\
$\mathrm{Zn}$ & 68 & 66 & 58 & 64.3 & 74 & - & 70 & 64 \\
$\mathrm{~Pb}$ & 0.5 & - & - & - & - & - & - & - \\
$\mathrm{Cu}$ & 100 & 108 & - & 96.5 & 98 & 78 & 104 & 108 \\
$\mathrm{Cr}$ & 405 & 425 & - & 368.1 & 409 & 418 & - & 370 \\
$\mathrm{~V}$ & - & - & - & 271.7 & 279 & 280 & - & 245 \\
$\mathrm{Sc}$ & 43 & 42 & - & - & - & - & - & - \\
$\mathrm{Nb}$ & - & - & 1.2 & 1.4 & - & - & - & - \\
$\mathrm{La}$ & 0.6 & 0.87 & - & - & - & - & - & - \\
$\mathrm{Ce}$ & 5.9 & 2.35 & - & - & - & - & - & - \\
$\mathrm{Nd}$ & - & 3.08 & - & - & - & - & - & - \\
$\mathrm{Sm}$ & 1.4 & 1.52 & - & - & - & - & - & - \\
$\mathrm{Eu}$ & 0.68 & 0.62 & - & - & - & - & - & - \\
$\mathrm{Gd}$ & - & 2.90 & - & - & - & - & - & - \\
$\mathrm{Tb}$ & 0.72 & 0.48 & - & - & - & - & - & - \\
$\mathrm{Ho}$ & - & 0.83 & - & - & - & - & - & - \\
$\mathrm{Tm}$ & - & 0.41 & - & - & - & - & - & - \\
$\mathrm{Yb}$ & 2.16 & 2.19 & - & - & - & - & - & - \\
$\mathrm{Lu}$ & 0.30 & 0.32 & - & - & - & - & - & - \\
\hline
\end{tabular}

Table 9. Trace elements in Sample IC II as reported.

\begin{tabular}{|c|c|c|c|c|c|c|c|c|}
\hline & VIG & UKAR & $\mathrm{COB}$ & WHOI & ALA & UGRE & IGOD & CSGS \\
\hline $\mathrm{Rb}$ & 5 & - & 1.13 & 2.0 & - & $<10$ & 3.7 & - \\
\hline $\mathrm{Sr}$ & 123 & 67 & 60 & 54.9 & - & 50 & 68 & 56 \\
\hline $\mathrm{Ba}$ & - & - & - & $<20$ & - & 12 & 50 & 1 \\
\hline $\mathrm{Li}$ & 7 & - & - & - & 10.3 & - & 6 & - \\
\hline $\mathbf{Y}$ & - & 28 & 28 & 29.0 & - & - & - & 25 \\
\hline $\mathrm{Zr}$ & - & 41 & 59 & 61.1 & - & - & - & 50 \\
\hline Co & 38 & 44 & - & 44.7 & 34.9 & 65 & 36 & 48 \\
\hline $\mathrm{Ni}$ & 83 & 91 & 95 & 102.8 & 93 & 128 & 79 & 118 \\
\hline $\mathrm{Zn}$ & 79 & 83 & 55 & 63.0 & 75 & - & 75 & 62 \\
\hline $\mathrm{Pb}$ & 0.5 & - & - & - & - & - & - & - \\
\hline $\mathrm{Cu}$ & 87 & 97 & - & 89.7 & 90 & 66 & 109 & 113 \\
\hline $\mathrm{Cr}$ & 374 & 377 & - & 297.9 & 356 & 346 & - & 319 \\
\hline v & - & 291 & - & 297.4 & 299 & 285 & - & 288 \\
\hline $\mathrm{Sc}$ & 46 & 47 & - & - & - & - & - & - \\
\hline $\mathrm{Nb}$ & - & - & 1 & 0.8 & - & - & - & - \\
\hline $\mathrm{La}$ & 2.15 & 1.15 & - & - & - & - & - & - \\
\hline $\mathrm{Ce}$ & 6.4 & 5.41 & - & - & - & - & - & - \\
\hline Nd & - & 4.07 & - & - & - & - & - & - \\
\hline $\mathrm{Sm}$ & 1.75 & 2.01 & - & - & - & - & - & - \\
\hline $\mathrm{Eu}$ & 0.68 & 0.81 & - & - & - & - & - & - \\
\hline Gd & - & 3.59 & - & - & - & - & - & - \\
\hline $\mathrm{Tb}$ & 0.85 & 0.65 & - & - & - & - & - & - \\
\hline Ho & - & 0.91 & - & - & - & - & - & - \\
\hline $\mathrm{Tm}$ & - & 0.4 & - & - & - & - & - & - \\
\hline $\mathrm{Yb}$ & 2.7 & 2.5 & - & - & - & - & - & - \\
\hline $\mathrm{Lu}$ & 0.49 & 0.46 & - & - & - & - & - & - \\
\hline
\end{tabular}

Table 10. Trace elements in Sample IC III as reported.

\begin{tabular}{lcccccccc}
\hline & VIG & UKAR & COB & WHOI & ALA & UGRE & IGOD & CSGS \\
\hline $\mathrm{Rb}$ & 7 & - & 1.07 & 1.9 & - & $<10$ & 3.7 & - \\
$\mathrm{Sr}$ & 100 & 69 & 65 & 56.6 & - & 51 & 69 & 57 \\
$\mathrm{Ba}$ & $\overline{7}$ & - & - & $<20$ & - & 11 & 18 & - \\
$\mathrm{Li}$ & $\overline{-}$ & 27 & $\overline{-}$ & $\overline{4}$ & 7.0 & - & 3.7 & - \\
$\mathrm{Y}$ & - & 24.2 & - & - & - & 20 \\
$\mathrm{Zr}$ & $\overline{-}$ & 35 & 49 & 55.5 & - & $\overline{-}$ & $\overline{-}$ & 41 \\
$\mathrm{Co}$ & 36 & 41 & - & 40.7 & 35.0 & 67 & 33 & 45 \\
$\mathrm{Ni}$ & 114 & 131 & 131 & 140.4 & 131 & 169 & 118 & 134 \\
$\mathrm{Zn}$ & 72 & 67 & 47.5 & 53.3 & 71 & - & 64 & 52 \\
$\mathrm{~Pb}$ & 0.5 & - & - & - & - & - & - & - \\
$\mathrm{Cu}$ & 217 & 86 & - & 82.8 & 82 & 59 & 88 & 99 \\
$\mathrm{Cr}$ & 434 & 462 & - & 356.8 & 415 & 425 & - & 386 \\
$\mathrm{~V}$ & - & 270 & - & 246.0 & 265 & 253 & - & - \\
$\mathrm{Sc}$ & 41 & - & - & - & - & - & - & - \\
$\mathrm{Nb}$ & - & - & 1.5 & 1.0 & - & - & - & - \\
$\mathrm{La}$ & 1.05 & 0.78 & - & - & - & - & - & - \\
$\mathrm{Ce}$ & 7.8 & 4.14 & - & - & - & - & - & - \\
$\mathrm{Nd}$ & - & 3.34 & - & - & - & - & - & - \\
$\mathrm{Sm}$ & 1.63 & 1.72 & - & - & - & - & - & - \\
$\mathrm{Eu}$ & 0.89 & 0.72 & - & - & - & - & - & - \\
$\mathrm{Gd}$ & - & 2.74 & - & - & - & - & - & - \\
$\mathrm{Tb}$ & 1.03 & 0.52 & - & - & - & - & - & - \\
$\mathrm{Ho}$ & - & 0.76 & - & - & - & - & - & - \\
$\mathrm{Tm}$ & - & 0.37 & - & - & - & - & - & - \\
$\mathrm{Yb}$ & 2.78 & 2.48 & - & - & - & - & - & - \\
$\mathrm{Lu}$ & 0.34 & 0.37 & - & - & - & - & - & - \\
\hline
\end{tabular}

